\title{
Avaliação de aspectos cognitivos em homens portadores de esquizofrenia em tratamento com haloperidol ou clozapina
}

\author{
Cognitive aspects evaluation of schizophrenic men under haloperidol or \\ clozapine treatment
}

Rejane Veiga Oliveira Johann e Cícero E. Vaz

\section{Resumo}

Objetivo: Avaliar, sob o ponto de vista psicológico, aspectos cognitivos de pacientes portadores de esquizofrenia associados ao tipo de medicamento antipsicótico em uso. Métodos: Participaram dois grupos: no grupo 1 os pacientes em tratamento com haloperidol $(n=14)$ e no 2 aqueles em tratamento com clozapina $(n=16)$. Ambos os grupos eram formados por indivíduos com esquizofrenia em tratamento psiquiátrico ambulatorial no Hospital de Clínicas de Porto Alegre (HCPA), cuja faixa etária variou entre 18 e 59 anos. Foram usados como instrumentos o Operational Checklist for Psychotic Illness (OPCRIT) para definição do diagnóstico de esquizofrenia e a técnica de Rorschach. Também se realizou a análise de variância (ANOVA) seguida do teste de Bonferroni, com nível de significância de $p \leq 0,05$ sobre os dados do teste de Rorschach. Resultados: 0 grupo 1 apresentou $\mathrm{F}+<80 \%$; FK $<\mathrm{KF}+\mathrm{K} ; \mathrm{FC}<\mathrm{CF}+\mathrm{C}$. $\mathrm{O}$ grupo 2 apresentou $\mathrm{F}+>80 \% ; \mathrm{FK}=\mathrm{KF}+\mathrm{K} ; \mathrm{FC}>\mathrm{CF}+\mathrm{C}$. Ambos os grupos apresentaram escore de forma ( $(F)$ na faixa clínica considerada normal. Conclusão: Os resultados do Rorschach permitem inferir que os pacientes medicados com clozapina apresentam indicativos de capacidade de produção $e$ adaptação à tarefa, controle pelo uso da inteligência sobre impulsos e instintos, capacidade para suportar frustração e funcionamento do raciocínio lógico em melhores condições do que os pacientes medicados com haloperidol.

Palavras-chave: Rorschach, esquizofrenia, medicamento antipsicótico.

\begin{abstract}
Objective: Evaluate from a psychological point of view cognitive aspects of schizophrenic patients, associated with the antipsychotic medicine type taken. Methods: The study analyzed three groups: group 1 patients medicated with haloperidol ( $n=14)$; group 2 - patients medicated with clozapine $(n=16)$. Groups 1 and 2 were schizophrenic men under psychiatric treatment at Hospital de Clínicas de Porto Alegre (HCPA). The age of all subjects ranged from 18 to 59 years. The following tools were used in the study: the Operational Checklist for Psychotic Illness (OPCRIT) to diagnose schizophrenia, and the Rorschach technique. The Rorschach data was subject to a variance analysis (ANOVA) followed by a Bonferroni test with a confidence interval of $p \leq 0.05$. Results: Group 1 presented $\mathrm{F}+<80 \% ; \mathrm{FK}<\mathrm{KF}+\mathrm{K} ; \mathrm{FC}<\mathrm{CF}+\mathrm{C}$. Group 2 presented $\mathrm{F}+>80 \% ; \mathrm{FK}=\mathrm{KF}+\mathrm{K} ; \mathrm{FC}>\mathrm{CF}+\mathrm{C}$. Both groups presented $\Sigma \mathrm{F}$ within the clinical range considered normal. Conclusion: The results show that patients medicated with clozapine present a relative capacity to participate on activities and tasks. Besides, they can control impulses and instincts by intellectual means in a superior fashion than patients medicated with haloperidol. Finally, it was also concluded that clozapine users present a greater ability to tolerate frustration, and a better general intellectual performance than haloperidol users.
\end{abstract}

Key words: Rorschach, schizophrenia, antipsychotic medication.

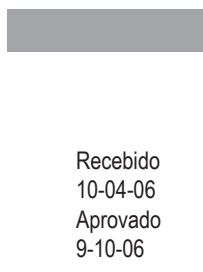

Aprovado

$9-10-06$

Pontifícia Universidade Católica do Rio Grande do Sul (PUCRS) (Johann RVO, Vaz CE) Universidade Luterana do Brasil (ULBRA) (Johann RVO) Conselho Nacional de Desenvolvimento Científico e Tecnológico (CNPq) (Vaz CE)

Artigo resultante da tese de doutorado intitulada Características de personalidade de homens portadores de esquizofrenia em tratamento com haloperidol ou clozapina. Apoio institucional da Coordenação de Aperfeiçoamento de Pessoal de Nível Superior (CAPES).

Correspondência para: Rejane Veiga Oliveira Johann

Avenida Cavalhada, 2.151/502A - Bairro Cavalhada - 91740-001 - Porto Alegre-RS - Tel.: (51) 3266-0004 e (51) 9901-8347 - e-mail: rejanejohann@terra.com.br 


\section{Introdução}

São muitas as pesquisas que, sob o ponto de vista psiquiátrico, buscam investigar as contribuições e contra-indicações da medicação antipsicótica de primeira ou de segunda geração para a recuperação de pacientes esquizofrênicos (Bechelli, 2000; Fleischhacker, 2002/2005; Frota, 2001; Nardi e Bueno, 2000; Louzã et al., 1999; Shirakawa et al., 2001; Stip, 2001).

Os antipsicóticos de primeira e/ou segunda geração reduzem os sintomas positivos da doença. Contudo, quando um grupo de pacientes faz uso da medicação de primeira geração, não obtém nenhuma melhora de seus sintomas ou, se ela ocorre, é apenas parcial. No entanto, mesmo esses pacientes refratários à medicação podem apresentar melhora se usarem a de segunda geração, especialmente se for a clozapina (Bechelli, 2000; Fleischhacker, 2002/2005). Os clínicos deviam pensar seriamente em oferecer clozapina e outros medicamentos antipsicóticos de segunda geração para pacientes que não respondem ou demonstram intolerância a antipsicóticos de primeira geração (Jackson et al., 2004).

Medicamento antipsicótico de primeira geração cuja descoberta de seus efeitos antipsicóticos ocorreu ao acaso, uma vez que foi sintetizado com a intenção de se obter um analgésico mais potente do que os existentes até então (Bechelli, 2000), ainda hoje o haloperidol é uma das drogas mais utilizadas no tratamento de pacientes esquizofrênicos.

O haloperidol é considerado uma droga de alta potência, pois, para atingir a mesma eficácia das drogas de baixa potência, suas doses devem ser até 20 vezes menores do que as menos potentes (Frota, 2001). Entretanto, apesar de apresentar efeitos colaterais autonômicos (sedação, hipotensão postural, etc.) menos proeminentes, ele apresenta maior possibilidade de desencadear reações extrapiramidais (distonia aguda, parkinsonismo medicamentoso, acatisia, síndrome neuroléptica maligna e discinesia tardia) do que os antipsicóticos de baixa potência (Bechelli, 2000), além de ser é considerado o agente causal mais freqüente da síndrome neuroléptica maligna (Hanel et al., 1998).

A clozapina foi o primeiro antipsicótico que demonstrou diferenças quantitativas e qualitativas em relação aos antipsicóticos de primeira geração. Ela demonstra vantagens não apenas no tratamento de pacientes refratários, mas também sobre os sintomas negativos da doença, mostrando-se eficiente em vários domínios psicossociais, incluindo a qualidade de vida e a prevenção de comportamentos suicidas (Fleischhacker, 2002/2005).

Em virtude do risco de causar agranulocitose (alteração hematológica), o uso da clozapina deve se restringir ao grupo de pacientes refratários e/ou intolerantes aos antipsicóticos de primeira geração (Bechelli, 2000; Graeff et al., 1999; Pádua, 2000; Rocha e Silva et al., 2001; Shirakawa et al., 2001; Stip, 2001). A agranulocitose causada pelo uso da clozapina é potencialmente fatal em cerca de $1 \%$ dos pacientes (Shirakawa et al., 2001). Os efeitos adversos mais comuns com o uso da clozapina são sonolência, salivação noturna, hipotensão postural e ganho de peso (Shirakawa et al., 2001).

Lamentavelmente não há antipsicóticos que não possuam efeitos colaterais inconvenientes. Portanto cautela, detecção precoce de efeitos adversos, diminuição de dose, substituição por outra droga, suspensão de associações desnecessárias e modificação comportamental são estratégias recomendadas (Abreu et al., 2000).

Testes psicológicos são freqüentemente aplicados em pacientes internados em hospitais psiquiátricos, buscando avaliar aqueles que apresentam potencial diagnóstico de esquizofrenia. Os testes mais utilizados são o de Rorschach, o teste de apercepção temática (TAT) e o Minnesota Multiphasic Personality Inventory (MMPI). O diagnóstico por meio de testes psicológicos é questionável, ponderam Lipton e Cancro (citados por Pull, 2002/2005). Há de se reconhecer que qualquer instrumento de avaliação do comportamento humano e dos processos cognitivos, incluindo entrevistas, tem sua margem de limitação.

Diversos autores (Adrados, 2000; Bohm, 1979; Klopfer e Kelly, 1977; Portuondo, 1976; Sousa, 1982; Vaz, 1997) apontam como sinais específicos de esquizofrenia no Rorschach a presença de respostas de forma pura (escore de forma $[\Sigma F]=$ $\mathrm{F}+, \mathrm{F}-, \mathrm{F} \pm$ ), ou seja, baixo controle geral sobre seus impulsos e instintos; baixo percentual de forma de boa qualidade $(\{F+x$ $100\} / \Sigma F)$ e, conseqüentemente, elevado percentual de respostas de forma de má qualidade (\{F- x 100/LF\}), o que denota imprecisão, incoerência e desorganização do raciocínio lógico. Além desses dados quantitativos, ratificando a desorganização do pensamento, é freqüente o aparecimento de fenômenos especiais como contaminação, crítica à técnica ou ao examinador e rejeição do cartão.

Em pesquisa para avaliação da capacidade de vinculação afetiva de pacientes esquizofrênicos do tipo paranóide, Veiga Oliveira e Vaz (2002) constataram indícios de respostas de movimento humano nos protocolos de Rorschach. Todavia essas respostas ocorreram em índice muito baixo no grupo de pacientes composto por homens, mas não houve respostas no formado por mulheres. Das respostas de movimento humano observadas, três foram de boa qualidade $(\mathrm{M}+)$, isto é, demonstraram ser pessoas com potencial criativo e alguma capacidade de empatia. A totalidade das respostas $\mathrm{M}+$ foi dada por pacientes que faziam uso de antipsicóticos de segunda geração, enquanto as duas respostas de movimento humano de má qualidade (M-) foram dos pacientes (homens) que usavam antipsicóticos de primeira geração. Quanto ao controle externo $(\mathrm{FC} \geq \mathrm{CF}+\mathrm{C})$, o grupo de homens avaliados apresentou $1 \mathrm{FC}<$ $0 \mathrm{CF}+4 \mathrm{C}$ e 0 de mulheres, $1 \mathrm{FC}<7 \mathrm{CF}+8 \mathrm{C}$, o que ratifica um maior descontrole emocional por parte das mulheres.

Pessoas portadoras de esquizofrenia apresentam precário controle interno $(\mathrm{M}<\mathrm{FM}+\mathrm{m})$, isto é, o movimento humano $(M \%)$ é menor do que a soma dos movimentos animal $(F M)$ e inanimado $(\mathrm{m})$. $\mathrm{O}$ controle externo $(\mathrm{FC}<\mathrm{CF}+\mathrm{C})$ se caracteriza pelo predomínio de cor com forma imprecisa (CF) e cor pura (C) sobre a cor com forma definida (FC), indicando descontrole emocional. Já o controle social raramente aparece, embora seja costumeiro haver respostas de claro-escuro (cor acromática) (FC', C'F, C'), representativas de angústia, ansiedade e depressão (Adrados, 1982).

Este estudo se propõe a avaliar, sob o ponto de vista psicológico, alguns aspectos cognitivos de pacientes esquizofrênicos associados ao tipo de medicamento antipsicótico em uso: haloperidol ou clozapina. 


\section{Métodos}

\section{Amostra}

A população incluída na amostra era constituída de homens com diagnóstico psiquiátrico prévio de esquizofrenia, com recidiva da doença, e em tratamento psiquiátrico no ambulatório de esquizofrenia do Hospital de Clínica de Porto Alegre (HCPA).

Foram escolhidos como amostra dois grupos. 0 grupo 1 (14 homens) era formado por pacientes diagnosticados como esquizofrênicos com recidiva da doença, e em tratamento farmacológico com haloperidol há, no mínimo, um ano e meio. Já o grupo 2 (16 homens) também reunia indivíduos com diagnóstico de esquizofrenia, recidiva da doença e em tratamento farmacológico há, no mínimo, um ano e meio, mas em uso de clozapina. $O$ estudo, com faixa etária variando entre 18 e 59 anos, adotou para a seleção da amostra os seguintes critérios de exclusão: diagnóstico cruzado de esquizofrenia com doenças afetivas, bem como pacientes que fazem uso concomitante de medicações de primeira e de segunda geração. Este último critério limitou bastante o tamanho da amostra do estudo, uma vez que é bastante comum o uso combinado de medicamentos de primeira e de segunda geração em pacientes esquizofrênicos.

\section{Instrumentos}

- Operational Checklist for Psychotic IIIness (OPCRIT): os pacientes foram avaliados previamente pela equipe do HCPA através do OPCRIT para definição do diagnóstico de esquizofrenia. Esse instrumento possibilita a definição de diagnósticos aproximados para pesquisas de transtornos mentais severos (Williams et al., 1996).

O OPCRIT é considerado um instrumento confiável, de rápida aplicação e de validade para pesquisas de doenças psiquiátricas (Craddock et al., 1996). Foi validado para o Brasil em trabalho conjunto da equipe do HCPA com a equipe de pesquisadores portugueses (Azevedo et al., 1999).

- Técnica de Rorschach: para avaliação do controle geral dos impulsos, do pensamento lógico, da percepção de conjunto, do senso de organização, da capacidade de discernimento, do senso de objetividade, da capacidade de análise, do senso de observação e da capacidade para tolerar frustrações, utilizou-se a técnica de Rorschach. Essa técnica, de autoria de Hermann Rorschach, teve sua primeira edição completa ao fim de junho de 1921. Trata-se de uma técnica que possibilita avaliar os elementos psicodinâmicos da pessoa, de modo abrangente e global, pois, por meio dela é possível avaliar a estrutura da personalidade do indivíduo e o funcionamento de seus psicodinamismos, seus traços de personalidade, o funcionamento de suas condições intelectuais, o nível de ansiedade básica e situacional, a depressão, suas condições afetivas e emocionais, o controle geral, a capacidade para suportar frustrações e conflitos, a integração humana, assim como possibilita avaliar os impulsos, os instintos e as reações emocionais (Vaz, 1997).

Existem vários sistemas para a classificação das respostas da técnica de Rorschach: Rorschach, Klopfer, Silveira, Ombredane, Canivet, Beck, Piotrowski, Hertz, Rappaport e Exner. Assim, a quantidade de sistemas hoje existentes nos dá a dimensão de como essa técnica foi, e continua sendo, muito utilizada e pesquisada. Todos os sistemas são capazes de chegar ao mesmo resultado na interpretação final acerca da avaliação de um protocolo de Rorschach. O sistema de classificação adotado neste estudo foi o de Klopfer adaptado por Vaz (1997) e usado pelos autores na prática clínica e de pesquisa.

Foram levados em conta os seguintes dados quantitativos da técnica de Rorschach:

- o número de respostas $(\mathrm{R})$ para avaliar a capacidade de produção e desempenho, bem como a adaptação à tarefa;

- $a$ incidência de respostas de forma pura $(\Sigma F \%)$ e de movimento animal (FM) para avaliação do controle geral e das reações impulsivas;

- a incidência e a qualidade das respostas de forma pura $(\mathrm{F}+\%, \mathrm{~F}-\%, \mathrm{~F} \pm \%)$ para avaliar o funcionamento do raciocínio lógico;

- a incidência de respostas de localização global (G), detalhes comuns (D) e detalhes incomuns (Dd) para avaliação da percepção de conjunto, do senso de organização, da capacidade de discernimento, do senso de objetividade, da capacidade de análise e do senso de observação;

- as respostas de sombreado de perspectiva e profundidade ( $F K, K F, K)$, cor cromática $(F C, C F, C)$ e forma de boa qualidade $(\mathrm{F}+)$ para avaliação da capacidade de frustração.

Foram feitas ilações na interpretação dinâmica com os índices encontrados na literatura como previsíveis (normais) das variáveis quantitativas.

\section{Procedimentos}

A Comissão Científica e a Comissão de Pesquisa e Ética em Saúde, reconhecida pela Comissão Nacional de Ética em Pesquisa (CONEP/MS) como Comitê de Ética em Pesquisa do HCPA e pelo Office for Human Research Protections (OHRP)/ United States Department of Health and Human Services (USDHHS), como Institutional Review Board (IRB000921), analisaram o projeto e aprovaram a realização dessa pesquisa.

A escolha dos pacientes para constituição da amostra se concretizou após a confirmação do diagnóstico pela equipe médica, com a aplicação do OPCRIT. Os pacientes foram convidados a participar da pesquisa, mas cada um assinou 0 seu termo de consentimento livre e esclarecido, assim como cada familiar responsável, e todos receberam uma cópia do documento.

Posteriormente foi realizada a aplicação da técnica de Rorschach nos dois grupos. A avaliação foi realizada de forma individual, em sala e em condições adequadas para essa finalidade, sendo a classificação das respostas de cada protocolo de Rorschach feita às cegas por integrantes do grupo de pesquisa treinados na técnica de Rorschach.

\section{Apresentação dos resultados}

Foi realizado estudo comparativo sobre os dados do Rorschach entre os grupos 1 (pacientes em tratamento com haloperidol) e 2 (pacientes em tratamento com clozapina), utilizando-se a análise de variância (ANOVA), seguida do teste de Bonferroni com nível de significância de $p \leq 0,05$. 
Os dados do Rorschach apontam para os resultados que se seguem.

- Os pacientes do grupo 1 (haloperidol) apresentaram um número bastante reduzido de respostas por protocolo $(R=9,71)$, visto que a faixa clínica considerada normal é de 15 a 30 respostas por protocolo. Já os do grupo 2 (clozapina) produziram em média 14,69 respostas por protocolo.

- O grupo 1 revelou um índice de respostas com $\mathrm{F}+$ inferior a $80 \%$ ( $\mathrm{F}+=72,77 \%)$, o que indica imprecisão, incoerência e desorganização do pensamento. Esse índice, associado ao aumento do número de respostas com índice de forma semidefinida ( $F \pm=21,88 \%$ ), indica que essas pessoas, quando abaladas emocionalmente, têm dificuldade em lidar com a situação e, portanto, perdem a qualidade do raciocínio. Embora não haja predominância de respostas de forma de má qualidade, a desorganização do pensamento desses pacientes aparece ratificada. 0 grupo 2, por sua vez, apresentou aumento de respostas de forma de boa qualidade $(\mathrm{F}+=86,34 \%)$ e conseqüente diminuição do índice de respostas de forma semidefinida ( $\mathrm{F} \pm=10,13 \%)$.

- Os dois grupos manifestaram um índice de respostas de detalhe comum (D) inferior à faixa clínica considerada normal, o que representa $40 \%$ a $55 \%$ das respostas, comprometendo assim a capacidade de discernimento e o senso de objetividade. 0 grupo 1 ( $D=13,29 \%$ ) está bem mais afastado da faixa clínica considerada normal do que o $2(20,83 \%)$.

- Existem diferenças entre os dois grupos quanto à capacidade para tolerar frustrações. Embora essas diferenças não sejam estatisticamente significavas, observa-se (Figura 1) que 0 grupo 1 não demonstra capacidade para tolerar frustrações, uma vez que não apresentou a proporção esperada em nenhuma das três variáveis do Rorschach que avaliam tal capacidade, enquanto o grupo 2 correspondeu às proporções da faixa clínica considerada normal em tais variáveis - grupo 1: $(F K<K F+K)=0,07<0,21$; $(F C<C F+C)=0,36<0,57$; grupo 2: $(F K=K F+K)=0,19=0,19$; $(\mathrm{FC}>\mathrm{CF}+\mathrm{C})=0,94>0,5)$.

- Os dois grupos apresentam indicadores de controle geral sobre impulsos e reações instintivas de acordo com a faixa clínica considerada normal, que seria o escore de forma $(\Sigma F)$ entre $30 \%$ e $50 \%$, não tendo sido constatada diferença estatisticamente significativa entre os grupos ( $\mathrm{G} 1=40,48 \mathrm{e}$ $\mathrm{G} 2=46,36)$.

\section{Figura 1}

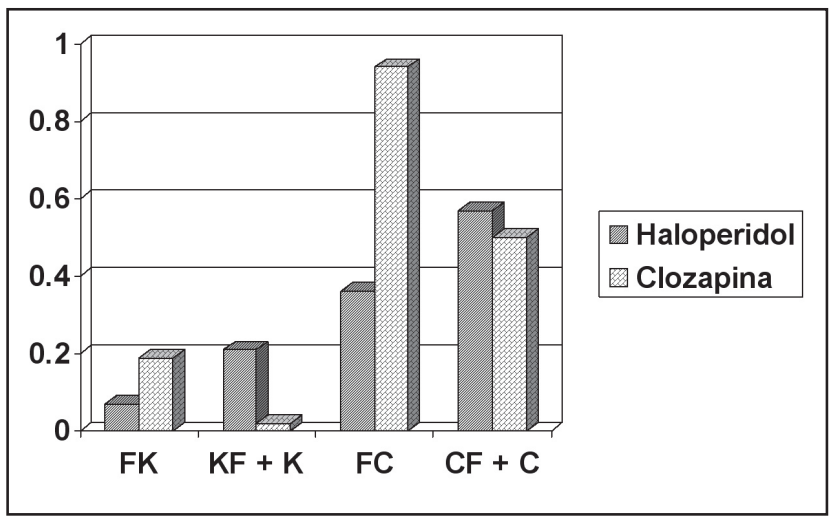

Quanto ao índice de respostas de movimento animal (FM), o grupo 1 apresentou em média uma resposta por protocolo, enquanto o grupo 2 apresentou 1,31 FM por protocolo.

- Os dois grupos apresentam alto índice de respostas de detalhes incomuns (Dd) - G1 $=22,76 \%$ e $\mathrm{G} 2=20,42 \%$ - uma vez que o máximo esperado seria de $17 \%$ do total de respostas por protocolo de Rorschach.

\section{Discussão dos resultados}

O número reduzido de respostas do grupo 1 (haloperidol) indica baixa capacidade de produção, desempenho e adaptação à tarefa. É comum aparecer um número reduzido de respostas no Rorschach em pessoas com defesas paranóides. Para evitar se expor, temendo a ameaça, o sujeito tenta se proteger através do controle intelectual sobre o meio externo e, desse modo, emite um baixo número de respostas. Já os pacientes do grupo 2 (clozapina) produzem respostas situadas na faixa clínica considerada normal, demonstrando ser adequada a sua capacidade de produção, desempenho e adaptação à tarefa.

Ao considerar que alogia, delírios e desorganização do pensamento são sintomas característicos de esquizofrenia, a literatura aponta como fato comum a presença no Rorschach de pacientes com esse diagnóstico, com baixo percentual de forma de boa qualidade $(F+)$ e elevado percentual de respostas de forma de má qualidade (F-), caracterizando raciocínio impreciso, incoerente e desorganizado (Adrados, 2000; Bohm, 1979; Klopfer e Kelly, 1977; Portuondo, 1976; Sousa, 1982; Vaz, 1997).

Um sujeito com raciocino lógico preciso, coerente, organizado e bem-ordenado apresenta como índice esperado $\mathrm{F}+$ $\geq 80 \%, \mathrm{~F} \pm$ até $20 \%$ e F- $=0 \%$, em relação ao total de respostas de forma pura ( $\Sigma F)$, conforme Vaz (1997). Neste estudo foram constatadas diferenças clínicas quanto ao funcionamento do raciocínio lógico entre os dois grupos.

Embora não tenham ocorrido diferenças estatisticamente significativas quanto à qualidade do determinante forma, se forem levados em consideração os índices apontados pela literatura como normais, pode-se dizer que a medicação com clozapina está ajudando na organização do raciocínio lógico desses pacientes, uma vez que eles apresentam raciocínio lógico preciso, coerente, organizado e bem-ordenado.

Os índices de respostas de detalhe comum (D) podem ser interpretados como comprometimento quanto à capacidade de discernimento e senso de objetividade dos pacientes tratados com haloperidol e com clozapina.

Uma pessoa com capacidade para tolerar frustrações apresenta respostas de sombreado perspectiva e profundidade, com forma clara e definida, em número maior ou igual ao sombreado perspectiva e profundidade com forma semidefinida $e$ sem forma ( $F K \geq K F+K)$, associado a respostas cromáticas, com forma clara e definida, em número maior ou igual à cor com forma semidefinida e sem forma ( $F C \geq C F+C)$, sendo, no mínimo, $80 \%$ das respostas de forma pura de boa qualidade $(\mathrm{F}+)$.

O baixo índice de respostas de movimento animal do grupo 1 (haloperidol), além de indicar que se trata de pessoas 
altamente defensivas, não-impulsivas e sem capacidade de iniciativa, quando associado à baixa incidência de respostas de forma de boa qualidade $(F+)$ e de detalhes comuns (D), também aponta para um sintoma comum da esquizofrenia: a avolição.

Os integrantes do grupo 2 (clozapina), embora também apresentem reduzido número de respostas de movimento animal e, portanto, igualmente possam ser vistos como pessoas altamente defensivas e não-impulsivas, denotam potencial para desenvolver capacidade de iniciativa porque apresentam adequados índices quanto ao número de respostas $(R)$ por protocolo de Rorschach, de somatório de forma $(\Sigma F)$ e de respostas de forma $(\mathrm{F}+)$ de boa qualidade.

Cabe destacar que o grupo 2, apesar de ter exibido um número de respostas de detalhe incomum (Dd) superior à faixa clínica considerada normal, apresentou adequados índices de respostas $(R)$ por protocolo de Rorschach e de respostas de forma de boa qualidade $(F+)$, e, considerando que os participantes desse grupo obtiveram baixo índice de respostas de detalhe comum (D), fica evidenciado que fazem uso de defesas obsessivas (Vaz, 1997). Já o grupo 1, além de revelar elevado índice de respostas $\mathrm{Dd}$, não apresenta os índices de $\mathrm{R}, \mathrm{F}+\mathrm{e}$ $D$ adequados, indicando comprometimento do funcionamento da inteligência pela percepção de minúcias em detrimento da capacidade de análise e do senso de observação.

A partir dos resultados encontrados nesta pesquisa e com base em aspectos da literatura, alguns dados que se destacaram podem auxiliar no entendimento de pacientes esquizofrênicos em tratamento com haloperidol e dos que são tratados com clozapina.

De modo geral, ao se analisar por meio da técnica de Rorschach o funcionamento da personalidade de pacientes com diagnóstico de esquizofrenia, observa-se baixa capacidade de produção e desempenho devido ao controle paranóide e ao inadequado controle geral sobre seus impulsos e instintos. São indivíduos propensos a perder o controle emocional e, conseqüentemente, suscetíveis a escapes agressivos. 0 raciocínio lógico deles é impreciso, incoerente e desorganizado e, sendo assim, apresentam prejuízo da capacidade de discernimento e do senso de objetividade, bem com não demonstram capacidade para tolerar frustrações.

\section{Referências}

Abreu PB, Bolognesi G, Rocha N. Prevenção e tratamento de efeitos adversos de antipsicóticos. Rev Bras Psiquiatr, 22(supl 1): 41-4, 2000.

Adrados I. Manual de psicodiagnóstico e diagnóstico diferencial. 2 ed. Petrópolis: Editora Vozes; 1982.

Adrados I. Teoria e prática do teste de Rorschach. 12 ed. Petrópolis: Editora Vozes; 2000.

Azevedo MH, Soares MJ, Coelho I, Dourado A, Valente J, Macedo A, Pato M, Pato C. Using consensus OPCRIT diagnoses: an efficient procedure for best-estimate lifetime diagnoses. Brit J Psychiatry, 175: 154-7, 1999.

Bechelli LPC. Impacto e evolução do emprego dos neurolépticos no tratamento da esquizofrenia. J Bras Psiq, 49(5): 131-47, 2000.

\section{Conclusão}

Os integrantes do grupo 1 (haloperidol) apresentaram no Rorschach baixa capacidade de produção, desempenho e adaptação à tarefa, típicos do uso de defesas paranóides, pois, para evitar se expor, temendo a ameaça, o sujeito tenta se proteger através do controle intelectual sobre o meio externo e, portanto, emite um baixo número de respostas. 0 pensamento lógico é impreciso, incoerente e desorganizado. Essas pessoas, quando abaladas emocionalmente, têm dificuldade em lidar com a situação e perdem a qualidade do raciocínio. Elas denotam maior comprometimento da capacidade de discernimento e do senso de objetividade, não demonstram capacidade para tolerar frustrações, justificando a avolição. Embora apresentem controle geral relativamente adequado sobre seus impulsos e instintos, são altamente defensivas, sem capacidade de iniciativa, e evidenciam demasiada preocupação com minúcias.

Os resultados da avaliação por meio da técnica de Rorschach aplicada no grupo 2 (clozapina), cujos pacientes foram tratados com medicação de segunda geração há pelo menos um ano e meio, demonstram que esses possuem adequada capacidade de produção, desempenho e adaptação à tarefa. Também revela que o raciocínio lógico está funcionando razoavelmente bem, que apresentam controle geral sobre seus impulsos e instintos, bem como certa capacidade para tolerar frustrações e potencial para desenvolver capacidade de iniciativa. A elevada preocupação com minúcias caracteriza 0 uso de defesas obsessivas.

Diante desses resultados pode-se inferir que o medicamento antipsicótico de primeira geração haloperidol está produzindo, parcialmente, o efeito esperado nos pacientes participantes do estudo no tocante à melhoria do controle geral sobre seus impulsos e instintos. Em contrapartida, os resultados apontam para melhor funcionamento geral da cognição dos pacientes tratados com clozapina, o que permite deduzir que esse medicamento antipsicótico de segunda geração é capaz de produzir mudanças no paciente em relação ao funcionamento geral da personalidade no que se refere a sua reintegração social.

Bohm E. Manual del psicodiagnóstico de Rorschach para psicólogos, médicos y pedagogos. 7 ed. Madrid: Ediciones Morata; 1979.

Bressan RA, Shirakawa I. Neurolépticos de ação prolongada e os avanços da psicofarmacologia da esquizofrenia. Psychiatry On-line Brazil Current Issues, 1(9): 1-7, 1996.

Craddock M, Asherson P, Owem MJ, Williams P, Farmer AF. Concurrent validity of the OPCRIT diagnostic system: comparison of OPCRIT diagnoses with consensus best-estimate lifetime diagnoses. J Bras Psiq, 169(1): 58-63, 1996.

Fleischhacker WW. Tratamento farmacológico da esquizofrenia: uma revisão. In: Maj M, Sartorius N. Esquizofrenia (trad.: Costa RC). 2 ed. Artmed Editora, p. 71-101, Porto Alegre, 2005. Trabalho original publicado em 2002. 
Frota LH. Cinqüenta anos de medicamentos antipsicóticos em psiquiatria: I fenotiazinas alifáticas. J Bras Psiq, 50(3-4): 121-41, 2001.

Gabbard GO. Psiquiatria psicodinâmica. 2 ed. Porto Alegre: Editora Artmed; 1998.

Graeff FG, Guimarães FS, Zuardi AW. Medicamentos antipsicóticos. In: Graeff FG, Guimarães FS. Fundamentos de psicofarmacologia. Editora Atheneu, p. 69-97, São Paulo, 1999.

Hanel RA, Sandmann MC, Kranich M, Bittencourt PRM. Síndrome neuroléptica maligna: relato de caso com recorrência associada ao uso de olanzapina. Arq Neuropsiquiatr, 56(4): 833-7, 1998.

Jackson CT, Covell NH, Essock SM. Differential effectiveness of clozapine for patients nonresponsive to or intolerant of first generation antipsychotic medications. Schizophrenia Bull, 30(2): 219-27, 2004.

Klopfer B, Kelly D. Técnica del psicodiagnóstico de Rorschach: método proyectivo para el diagnóstico de la personalidad. Buenos Aires: Editorial Paidós; 1977.

Louzã Neto MR, Shirakawa I, Barros L. Esquizofrenia: dois enfoques complementares. São Paulo: Lemos Editorial; 1999.

Nardi AE, Bueno JR Esquizofrenia: avanços no tratamento. In: Bueno JR, Nardi AE (orgs.). Diagnóstico e tratamento em psiquiatria. Rio de Janeiro: Editora Medsi, p. 171-218, 2000.

Pádua AC. Esquizofrenia. In: Cordioli AV et al. Psicofármacos: consulta rápida. 2 ed. Porto Alegre: Editora Artes Médicas Sul; 2000.
Portuondo JA. El psicodiagnóstico de Rorschach en psicología clínica. 2 ed. Madrid: Biblioteca Nueva;1976.

Pull CB. Diagnóstico da esquizofrenia: uma revisão. In: Maj M, Sartorius N. Esquizofrenia (trad.: Costa RC). 2 ed. Editora Artmed, p. 13-41, Porto Alegre, 2005. Trabalho original publicado em 2002.

Rocha e Silva CE, Pereira BB, Rozenthal M, Elkis H. Estudo-piloto com clozapina em hospital público: resultados de um ano de acompanhamento. Rev Bras Psiq, 23(4): 180-7, 2001.

Shirakawa, I, Bressan RA, Chaves AC. Tratamento farmacológico da esquizofrenia. In: Shirakawa I, Chaves AC, Mari J. O desafio da esquizofrenia. Lemos Editorial, p. 135-48, São Paulo, 2001.

Sousa CC. 0 método de Rorschach. 3 ed. São Paulo: Editoria da Universidade de São Paulo; 1982.

Stip E. Antipsicóticos nuevos: temas y controversias. Tipicidad de los antipsicóticos atípicos. Rev Psiq del Uruguay, 65(1): 70-91, 2001.

Vaz CE. O Rorschach. Teoria e desempenho. 3 ed. São Paulo: Editora Manole; 1997.

Veiga Oliveira R, Vaz CE. Capacidade de estabelecer vínculo afetivo em pacientes com esquizofrenia tipo paranóide. In: Vaz CE, Veit CA (orgs.). Personalidade, cultura e técnicas projetivas. Edipucrs, $p$. 169-2, Porto Alegre, 2002.

Williams J, Farmer AE, Ackenheil M, Kaufmann CA, McGuffin P. A multicentre inter-rater reliability study using the OPCRIT computerized diagnostic system. Psych Med, 26(4): 775-83, 1996. 